\title{
A cost-effective synthesis of enantiopure ovothiol A from L-histidine, its natural precursor
}

\author{
Arash Mirzahosseini, ${ }^{a, b}$ Sándor Hosztafi, ${ }^{a, b}$ Gergő Tóth, ${ }^{a, b}$ and Béla Noszál ${ }^{a, b} *$ \\ ${ }^{a}$ Department of Pharmaceutical Chemistry, Semmelweis University, Högyes Endre utca 9. \\ Budapest, 1092, Hungary \\ ${ }^{b}$ Research Group of Drugs of Abuse and Doping Agents, Hungarian Academy of Sciences, \\ Széchenyi István tér 9., Budapest, 1051, Hungary \\ E-mail: noszal.bela@pharma.semmelweis-univ.hu
}

DOI: http://dx.doi.org/10.3998/ark.5550190.p008.872

\begin{abstract}
Ovothiol A is a naturally occurring 4-mercaptohistidine derivative, and one of the most potent thiol-containing antioxidants. The synthesis of ovothiol A was elaborated by introducing the thiol group onto protected 3-methyl-L-histidine via halogenation and Ullmann reaction. This enantiopure synthesis of ovothiol A, which uses L-histidine as starting material, requires only five steps, and compared to prior synthesis, is both cost and time effiecent.
\end{abstract}

Keywords: Ovothiol, antioxidant, trypanosomatid, histidine

\section{Introduction}

Ovothiol A [(2S)-2-amino-3-(1-methyl-4-sulfanyl-1H-imidazol-5-yl)propanoic acid] is a naturally occurring 4-mercaptohistidine derivative ${ }^{1-3}$ first observed in sea urchin (Strongylocentrotus purpuratus) eggs by Turner et al. ${ }^{4}$ Concerning its molecular properties, ovothiol $\mathrm{A}$ is a chemical entity in a class of its own with an extremely high number and variety of functional groups for its molecular weight, and its thiolate basicity being by far the lowest one among all molecules reported. ${ }^{5,6}$ Ovothiols are considered to be the most potent natural antioxidants, ${ }^{7,8}$ presumably due to their low thiolate basicity and the concomitant ready oxidizability. They act as protective antioxidants ${ }^{9,10}$ in many parasitic protozoa. ${ }^{11-15}$ These peculiarities have attracted pharmaceutical interest to create prospective antitrypanosomal chemotherapeutic agents. ${ }^{16,17}$ Despite this, the commercial availability of ovothiol A is limited, and its isolation from marine $\operatorname{algae}^{10}$ and sea urchin eggs $^{4}$ is a troublesome task. Total syntheses ${ }^{18-21}$ have been elaborated (Scheme 1) and are the favored method to obtain ovothiol A. 


$$
\stackrel{\mathrm{NaCN}+\mathrm{CH}_{2} \mathrm{O}}{\stackrel{\mathrm{Me}-\mathrm{NH}_{3}{ }^{+} \mathrm{Cl}^{-}}{\longrightarrow} \stackrel{\mathrm{HCO}}{\longrightarrow} \mathrm{H}_{2} \mathrm{~S} / \mathrm{Ac}_{2} \mathrm{O}}
$$<smiles></smiles><smiles>CO[N+](C)(O)COc1cn(C)c(CO)c1C=O</smiles><smiles>CCN(C)[Si](C)(C)Cl</smiles><smiles>COc1ccc(CSc2cn(C)cn2)cc1</smiles><smiles>CCOC(=O)OCC</smiles>

$\mathrm{NaBH}_{4}$<smiles>COc1ccc(CCl)cc1</smiles><smiles>COc1ccc(CSc2ncn(C)c2C=O)cc1</smiles><smiles>COc1ccc(CSc2ncn(C)c2CO)cc1</smiles><smiles>CCOC1=NC(C(C)C)C(OCC)=NC1Cc1c(SCc2ccc(OC)cc2)ncn1C</smiles><smiles>COc1ccc(CSc2ncn(C)c2CC(N)C(=O)O)cc1</smiles>

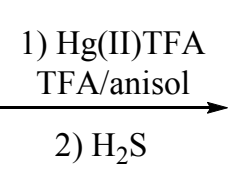

$\mathrm{Me}$<smiles></smiles>

6

Scheme 1. The total syntheses routes proposed by Holler et al. ${ }^{18,19}$ (left) and Ohba et al. ${ }^{20,21}$ (right). ${ }^{a}(R)$-2,5-Dihydro-3,6-diethoxy-2-isopropylpyrazine, $n$-butyllithium. 
Holler et al., ${ }^{19}$ found that $N$ - $\alpha$-benzoyl-4-bromo-1-methylhistidine did not undergo halogensulfur substitution with $t$-butanethiolate. Therefore, they assembled the thioimidazole moiety through the reaction of $N$-(cyanomethyl)- $N$-methylformamide and hydrogen sulfide to form the C-S bond. Subsequently, the amino acid side chain could easily be built enantioselectively using formaldehyde and thionyl chloride to introduce a chloromethyl group on the imidazole ring, followed by the $n$-butyllithium mediated reaction with (3R)-2,5-diethoxy-3,6-dihydro-3isopropylpiperazine. Ohba et al., ${ }^{21}$ overcame the direct bromine-sulfur displacement on 4-bromo1-methyl- $1 \mathrm{H}$-imidazole-5-carbaldehyde, prior to assembling the histidine side-chain (using the same procedure as Holler et al.). Ohba et al. formed 4-bromo-1-methyl-1H-imidazole-5carbaldehyde by brominating imidazole, followed by methylation with methyl iodide. The $\mathrm{Br}-\mathrm{S}$ substitution was carried out with thiophenol, (4-tolyl)methanethiol, and 1-naphthalenethiol.

Herein we report a new, simple synthesis that combines the assets of the earlier total syntheses. By using L-histidine as starting material, containing the desired stereogenic center, ovothiol A has been produced in an enantiopure, convenient and cost-effective way.

\section{Results and Discussion}

For the synthesis of ovothiol A, the natural precursor was the obvious starting molecule of choice. To introduce a thiol functional group onto L-histidine at C5 we applied a new approach alongside those already investigated by Holler et al. ${ }^{19}$ The halogenation of a protected 3-methylL-histidine, followed by substitution with sulfur using a modified Ullmann reaction was attempted (Scheme 2). For this purpose first the nitrogen atoms were protected: L-histidine was converted to $2(92 \%)$, using similar reaction conditions as those described by Abdo et al. ${ }^{22}$ During the reaction of L-histidine with di-tert-butyl dicarbonate the $\alpha$-amino and $\tau$-nitrogen groups of L-histidine are protected (this is the major product as opposed to $\pi$-nitrogen protected L-histidine, due to the sterically hindered $\pi$ position for the bulky Boc group). Next, the carboxyl group was protected by esterification (due to incompatibility of the carboxyl with bromination ${ }^{23}$ ), while simultaneous methylation of the nitrogen 3 atom was achieved with methyl iodide. Parallel to the methylation of the $\pi$-nitrogen the Boc protecting group on the imidazole ring was removed resulting in $3(83 \%)$ contaminated by the dimethylated quaternary salt, which was separated by column chromatography. The reaction sequence suggested by Van Den Berge and Robiette ${ }^{24}$ for $\mathrm{N}$-methylation of imidazole compounds was also attempted: however, better reaction yields were not achieved for this particular case. The resulting protected 3-methyl-L-histidine derivative was subsequently reacted with elemental bromine (in aqueous conditions, $0{ }^{\circ} \mathrm{C}$ ) ${ }^{23}(66 \%$ ), and alternatively with $\mathrm{N}$-bromosuccinimide ${ }^{25,26}(75 \%)$. The latter method was favored, as it seemed to produce less dibrominated side product. Worthy of note was that, even though compounds 2 and $\mathbf{3}$ are commercially available, the synthetic route described here is more cost-effective, not only compared to previously described ovothiol total syntheses, but even if compound $\mathbf{3}$ were used as starting material from commercial sources. The bromo-derivative $\mathbf{4}$ was then reacted 
with (4-tolyl)methanethiol, to substitute the bromine atom with sulfur, thus introducing a thioether group (S-protected ovothiol). This reaction step was optimized in terms of solvent (acetonitrile/DMF), base (sodium hydride/potassium tert-butoxide), temperature $\left(80-120{ }^{\circ} \mathrm{C}\right.$ only for DMF), and reaction time (3, 6, 12 and $24 \mathrm{~h}$ ). Since, the solubility of the reactant 4 was very poor in acetonitrile, no reaction took place and therefore DMF was used. Sodium hydride produced higher yields than potassium tert-butoxide, and the reaction was practically complete in 3 hours. The reaction only proceeded at $120^{\circ} \mathrm{C}$. The configurational stability of $\mathbf{4}$ during the reaction with $\mathrm{NaH}$ at $120{ }^{\circ} \mathrm{C}$ was confimed with the ${ }^{1} \mathrm{H}$ NMR spectrum of $\mathbf{5}$, which did not show split peaks for the alpha proton. Upon addition of copper(I) iodide catalyst the reaction yield dropped to almost $0 \%$ (determined by HPLC-MS) and a deep purple colored cloudy solution was formed. Since histidine, through its imidazole ring, is known for being a good chelator of transition metals, L-histidine in DMF was reacted with $\mathrm{CuI}$ which resulted in a similar dark purple/brown color, indicating that some sort of stable complex was being produced; therefore $\mathrm{CuI}$ could not be used as a catalyst. Thus, $\mathbf{4}$ was reacted with excess (4-tolyl)methanethiol using sodium hydride in DMF to yield the $N$ - $\alpha$ and $S$-protected derivative of ovothiol 5 (74\%). The resulting product 5, was deprotected to afford L-ovothiol A $\mathbf{6}$, using mercury(II) trifluoroacetate in trifluoroacetic acid (the latter simultaneously cleaves the Boc group) (78\%), as described previously by Holler et al. ${ }^{19}$

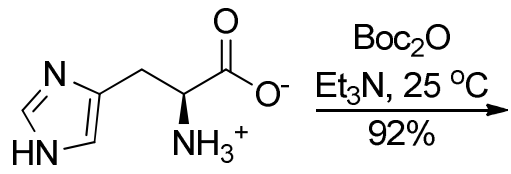

1<smiles>CC(C)(C)OC(=O)NC(Cc1cn(C(C)(C)C)cn1)C(=O)O</smiles>

2<smiles>COC(=O)NC(Cc1cncn1C)C(=O)OC</smiles>

3<smiles>COC(=O)C(Cc1c(Br)ncn1C)NC(=O)OCCCCCOC(C)(C)C</smiles>

4<smiles></smiles>

6

Scheme 2. The synthesis route of L-ovothiol A<smiles>Cc1ccc(CSc2ncn(C)c2CC(NC(=O)O)C(=O)O)cc1</smiles> 
Since, L-ovothiol A is an extremely unstable thiol, readily oxidized by air, the only literature procedure for assigning the correct configuration is to measure the specific rotation of the more stable disulfide derived from biological extractions. To characterize the configuration of the endproduct, an aqueous solution of L-ovothiol A was allowed to be oxidized by atmospheric oxygen (Scheme 3). The stable disulfide 7 was identical with the disulfide of naturally derived Lovothiol A with regard to the ${ }^{1} \mathrm{H}$ NMR, UV spectrum and specific rotation. ${ }^{2,19}$ Furthermore, mercury contamination levels were examined in our end-product as suggested by the European Pharmacopoeia; ${ }^{27}$ the heavy metal impurity level being less than $10 \mathrm{ppm}$ in L-ovothiol A disulfide tetra(trifluoroacetate) 7 .
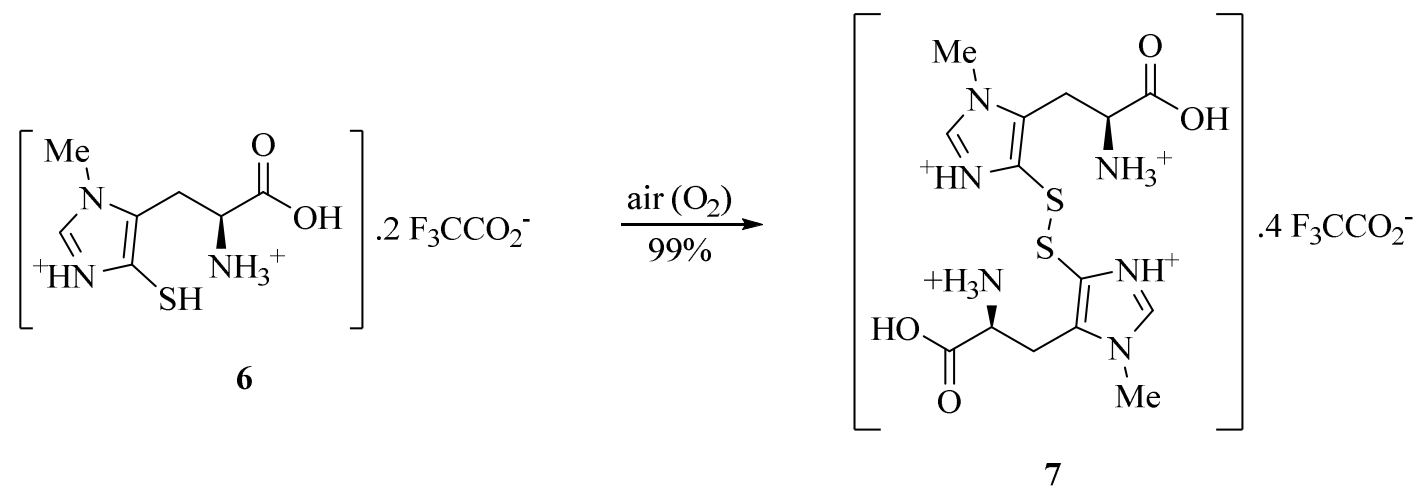

Scheme 3. The oxidation of L-ovothiol A

\section{Conclusions}

A short synthesis of enantiopure ovothiol A is described, which is cost efficient and produces the highly potent antioxidant in adequate quantities for biological assays.

\section{Experimental Section}

General. L-Histidine was purchased from Bachem. All other chemicals were purchased from Sigma and were used without further purification. Column chromatography was carried out by using Merck silica gel 60, 0.063-0.200 mm (70-230 mesh ASTM). Melting points were determined with OptiMelt MPA100, SRS. NMR measurements were recorded on a Varian 600 $\mathrm{MHz}$ spectrometer at $25{ }^{\circ} \mathrm{C}$. The chemical shift values reported are downfield from an internal reference, DSS in $\mathrm{D}_{2} \mathrm{O}$, and TMS in all other solvents. The exact mass of the synthesized and isolated compounds was determined with an Agilent 6230 time-of-flight mass spectrometer equipped with a JetStream electrospray ion source in positive ion mode. JetStream parameters: drying gas $\left(\mathrm{N}_{2}\right)$ flow and temperature: $10.0 \mathrm{~L} / \mathrm{min}$ and $325{ }^{\circ} \mathrm{C}$; nebulizer gas $\left(\mathrm{N}_{2}\right)$ pressure: 10 
psi; capillary voltage: $4000 \mathrm{~V}$; sheath gas flow and temperature: $325^{\circ} \mathrm{C}$ and $7.5 \mathrm{~L} / \mathrm{min}$. TOF MS parameters: fragmentor voltage: $170 \mathrm{~V}$; skimmer potential: $170 \mathrm{~V}$; OCT $1 \mathrm{RF}$ Vpp: $750 \mathrm{~V}$. Samples were introduced $(0.1-0.3 \mu \mathrm{L})$ by the Agilent 1260 Infinity HPLC system (flow rate 0.5 $\mathrm{mL} / \mathrm{min}, 70 \%$ methanol/water mixture $0.1 \%$ formic acid). Reference masses of $\mathrm{m} / \mathrm{z} 121.050873$ and 922.009798 were used to calibrate the mass axis during analysis. Mass spectra were acquired over the $\mathrm{m} / \mathrm{z}$ range $100-1000$ at an acquisition rate of $250 \mathrm{~ms} / \mathrm{spectrum}$ and processed using Agilent MassHunter B.02.00 software. Specific rotations were measured on a Jasco Model P2000 polarimeter at the $\mathrm{D}$ line of sodium.

\section{(2S)-2-[(tert-Butoxycarbonyl)amino]-3-[1-(tert-butoxycarbonyl)-1H-imidazol-4-yl]-} propanoic acid (2). To a suspension of L-histidine 1 (1.43 g, $9.2 \mathrm{mmol})$ in $\mathrm{MeOH}(30 \mathrm{~mL})$, ditert-butyl dicarbonate $(3.24 \mathrm{~g}, 21.2 \mathrm{mmol})$ and $\mathrm{Et}_{3} \mathrm{~N}(1.8 \mathrm{~mL}, 18.4 \mathrm{mmol})$ were added. The reaction mixture was stirred at room temperature overnight. The reaction mixture was then concentrated under vacuum and the crude product was purified on silica gel with column chromatography $\left(\mathrm{CHCl}_{3} / \mathrm{MeOH}, 9: 1\right)$ to give the title compound $2(3.01 \mathrm{~g}, 92 \%)$ as a colorless solid: mp 73-75 ${ }^{\circ} \mathrm{C}^{22}$ (from $\mathrm{CHCl}_{3}$ ); ${ }^{1} \mathrm{H}$ NMR (600 MHz, DMSO- $d_{6}$ ): $\delta_{\mathrm{H}} 1.40(9 \mathrm{H}, \mathrm{s}$, $\left.\mathrm{N}^{\alpha} \mathrm{CO}_{2} \mathrm{C}\left(\mathrm{CH}_{3}\right)_{3}\right), 1.61\left(9 \mathrm{H}, \mathrm{s}, \mathrm{N}^{\mathrm{Im}} \mathrm{CO}_{2} \mathrm{C}\left(\mathrm{CH}_{3}\right)_{3}\right), 2.87(1 \mathrm{H}, \mathrm{dd}, J 8,15 \mathrm{~Hz}, \beta \mathrm{H}), 3.08(1 \mathrm{H}, \mathrm{dd}, J$, $15 \mathrm{~Hz}, \beta \mathrm{H}), 4.25(1 \mathrm{H}, \mathrm{dd}, J 4,8 \mathrm{~Hz}, \alpha \mathrm{H}), 7.29\left(1 \mathrm{H}, \mathrm{s}, \operatorname{Im}^{5} \mathrm{H}\right), 8.06\left(1 \mathrm{H}, \mathrm{s}, \mathrm{Im}^{2} \mathrm{H}\right) ;{ }^{13} \mathrm{C}$ NMR $(600$ $\left.\mathrm{MHz}, \mathrm{CD}_{3} \mathrm{OD}\right): \delta_{\mathrm{C}} 28.1\left(\mathrm{~N}^{\alpha} \mathrm{Boc}-\mathrm{CH}_{3}\right), 28.8\left(\mathrm{~N}^{\mathrm{Im}} \mathrm{Boc}_{-} \mathrm{CH}_{3}\right), 31.1(\beta \mathrm{C}), 56.6(\alpha \mathrm{C}), 80.1\left(\mathrm{~N}^{\alpha} \mathrm{Boc}-t-\right.$ C), $86.9\left(\mathrm{~N}^{\mathrm{Im}}\right.$ Boc- $\left.t-\mathrm{C}\right), 116.1\left(\mathrm{Im}^{4} \mathrm{C}\right), 137.6\left(\mathrm{Im}^{5} \mathrm{C}\right), 141.1\left(\mathrm{Im}^{2} \mathrm{C}\right), 148.3\left(\mathrm{~N}^{\alpha}\right.$ Boc-C $\left.=\mathrm{O}\right), 157.5$ $\left(\mathrm{N}^{\mathrm{Im}}\right.$ Boc-C=O), $178.1\left(\mathrm{CO}_{2} \mathrm{H}\right)$; HRMS $m / z[\mathrm{M}+\mathrm{H}]^{+}$Calcd: 356.1822, Found: 356.1824 .

Methyl (2S)-2-[(tert-butoxycarbonyl)amino]-3-(1-methyl-1H-imidazol-5-yl)propanoate (3). To a solution of $2(3.01 \mathrm{~g}, 8.5 \mathrm{mmol})$ in $\mathrm{MeOH}(20 \mathrm{~mL})$ at $0{ }^{\circ} \mathrm{C}$, was added $\mathrm{MeI}(1.32 \mathrm{~mL}, 21.3$ $\mathrm{mmol}$ ). The reaction mixture was allowed to stir overnight at $0{ }^{\circ} \mathrm{C}$ and then extracted (EtOAc) (7 $<\mathrm{pH}<8)$ and washed with water. The resulting organic layer was dried $\left(\mathrm{Na}_{2} \mathrm{SO}_{4}\right)$, filtered and concentrated under vacuum. The crude product was purified on silica gel with column chromatography (EtOAc/acetone, 9:1) to give the title compound $\mathbf{3}(1.97 \mathrm{~g}, 83 \%)$ as a colorless solid (the position of the methyl group on the imidazole ring was confirmed using NOESY ${ }^{28}$ and $\left.{ }^{1} \mathrm{H}_{-}{ }^{15} \mathrm{~N} \mathrm{HMBC}{ }^{29}\right)$ : mp 109-110 ${ }^{\circ} \mathrm{C}$ (from EtOAc); ${ }^{1} \mathrm{H}$ NMR $\left(600 \mathrm{MHz}, \mathrm{CD}_{3} \mathrm{OD}\right): \delta_{\mathrm{H}} 1.40(9 \mathrm{H}, \mathrm{s}$, $\left.\mathrm{CO}_{2} \mathrm{C}\left(\mathrm{CH}_{3}\right)_{3}\right), 2.98(1 \mathrm{H}, \mathrm{dd}, J 9,15 \mathrm{~Hz}, \beta \mathrm{H}), 3.14(1 \mathrm{H}, \mathrm{dd}, J 5,15 \mathrm{~Hz}, \beta \mathrm{H}), 3.66\left(3 \mathrm{H}, \mathrm{s}, \mathrm{NCH}_{3}\right)$, $3.74\left(3 \mathrm{H}, \mathrm{s}, \mathrm{OCH}_{3}\right), 4.42(1 \mathrm{H}, \mathrm{dd}, J 5,9 \mathrm{~Hz}, \alpha \mathrm{H}), 6.78\left(1 \mathrm{H}, \mathrm{s}, \mathrm{Im}^{5} \mathrm{H}\right), 7.59\left(1 \mathrm{H}, \mathrm{s}, \mathrm{Im}^{2} \mathrm{H}\right) ;{ }^{13} \mathrm{C}$ NMR (600 MHz, CD $\left.\mathrm{CD}_{3} \mathrm{OD}\right): \delta_{\mathrm{C}} 27.1(\beta \mathrm{C}), 28.7\left(\mathrm{Boc}_{-} \mathrm{CH}_{3}\right), 31.8\left(\mathrm{NCH}_{3}\right), 52.9\left(\mathrm{OCH}_{3}\right), 54.2(\alpha \mathrm{C})$, 80.8 (Boc-t-C), $127.5\left(\mathrm{Im}^{5} \mathrm{C}\right), 129.4\left(\mathrm{Im}^{4} \mathrm{C}\right), 139.2\left(\mathrm{Im}^{2} \mathrm{C}\right), 157.7(\mathrm{Boc}-\mathrm{C}=\mathrm{O}), 173.4\left(\mathrm{CO}_{2}\right)$; HRMS $m / z[\mathrm{M}+\mathrm{H}]^{+}$Calcd: 284.1610, Found: 284.1602.

Methyl (2S)-3-(4-bromo-1-methyl-1H-imidazol-5-yl)-2-[(tert-butoxycarbonyl)amino]propanoate (4). To a solution of $3(1.02 \mathrm{~g}, 3.6 \mathrm{mmol})$ in $\mathrm{MeOH}(10 \mathrm{~mL})$ at $0{ }^{\circ} \mathrm{C}$, was added in small portions $\mathrm{N}$-bromosuccinimide $(0.71 \mathrm{~g}, 4.0 \mathrm{mmol})$. The reaction mixture was stirred at $0{ }^{\circ} \mathrm{C}$ for 2 hours, and then the reaction mixture was concentrated under vacuum. The crude product was purified on silica gel with column chromatography (EtOAc/ $\left.\mathrm{CHCl}_{3}, 9: 1\right)$ to give the title compound $4(0.98 \mathrm{~g}, 75 \%)$ as a colorless glassy solid: ${ }^{1} \mathrm{H}$ NMR $\left(600 \mathrm{MHz}, \mathrm{CD}_{3} \mathrm{OD}\right): \delta_{\mathrm{H}} 1.38$ 
$\left(9 \mathrm{H}, \mathrm{s}, \mathrm{CO}_{2} \mathrm{C}\left(\mathrm{CH}_{3}\right)_{3}\right), 2.99(1 \mathrm{H}, \mathrm{dd}, J 9,15 \mathrm{~Hz}, \beta \mathrm{H}), 3.15(1 \mathrm{H}, \mathrm{dd}, J 6,15 \mathrm{~Hz}, \beta \mathrm{H}), 3.69(3 \mathrm{H}, \mathrm{s}$, $\left.\mathrm{NCH}_{3}\right), 3.73\left(3 \mathrm{H}, \mathrm{s}, \mathrm{OCH}_{3}\right), 4.40(1 \mathrm{H}, \mathrm{t}, J 7 \mathrm{~Hz}, \alpha \mathrm{H}), 7.55\left(1 \mathrm{H}, \mathrm{s}, \mathrm{Im}^{2} \mathrm{H}\right) ;{ }^{13} \mathrm{C} \mathrm{NMR}(600 \mathrm{MHz}$, $\left.\mathrm{CD}_{3} \mathrm{OD}\right): \delta_{\mathrm{C}} 27.1\left(\mathrm{Boc}_{-} \mathrm{CH}_{3}\right), 28.6(\beta \mathrm{C}), 33.0\left(\mathrm{NCH}_{3}\right), 49.8\left(\mathrm{OCH}_{3}\right), 53.7(\alpha \mathrm{C}), 79.4(\mathrm{Boc}-t-\mathrm{C})$, $115.5\left(\mathrm{Im}^{5} \mathrm{C}\right), 126.8\left(\mathrm{Im}^{4} \mathrm{C}\right), 138.9\left(\mathrm{Im}^{2} \mathrm{C}\right), 157.4(\mathrm{Boc}-\mathrm{C}=\mathrm{O}), 181.5\left(\mathrm{CO}_{2}\right) ; \mathrm{HRMS} \mathrm{m} / \mathrm{z}[\mathrm{M}+\mathrm{H}]^{+}$ Calcd: 362.0715, Found: 362.0720.

(2S)-2-[(tert-Butoxycarbonyl)amino]-3-\{1-methyl-4-[(4-methylbenzyl)sulfanyl]-1Himidazol-5-yl\}propanoic acid (5). To a stirred solution of $\mathrm{NaH}(98 \mathrm{mg}, 4.1 \mathrm{mmol})$ in dry DMF $(10 \mathrm{~mL})$ under an $\mathrm{N}_{2}$ atmosphere at room temperature was added dropwise a solution of 4 (0.98 $\mathrm{g}, 2.7 \mathrm{mmol})$ and (4-tolyl)methanethiol $(1 \mathrm{~mL}, 8.1 \mathrm{mmol})$ in dry DMF $(10 \mathrm{~mL})$. The reaction mixture was then heated at $120{ }^{\circ} \mathrm{C}$ under an atmosphere of $\mathrm{N}_{2}$ for $3 \mathrm{~h}$. After cooling, the reaction mixture was concentrated under vacuum to leave a brown oil. The crude product was purified on silica gel with column chromatography $\left(\mathrm{CH}_{2} \mathrm{Cl}_{2} / n\right.$-hexane, $\left.2: 1\right)$ to give the title compound $\mathbf{5}$

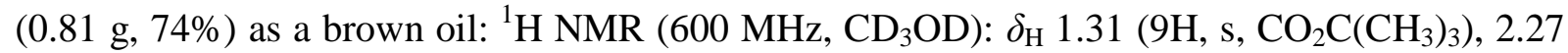
$\left(3 \mathrm{H}, \mathrm{s}, \mathrm{ArCH}_{3}\right), 2.63(1 \mathrm{H}, \mathrm{dd}, J 10,15 \mathrm{~Hz}, \beta \mathrm{H}), 2.82(1 \mathrm{H}, \mathrm{dd}, J 4,15 \mathrm{~Hz}, \beta \mathrm{H}), 3.67(3 \mathrm{H}, \mathrm{s}$, $\left.\mathrm{NCH}_{3}\right), 3.81\left(1 \mathrm{H}, \mathrm{d}, J 13 \mathrm{~Hz}, \mathrm{SCH}_{2}\right), 3.86\left(1 \mathrm{H}, \mathrm{d}, J 13 \mathrm{~Hz}, \mathrm{SCH}_{2}\right), 4.09(1 \mathrm{H}, \mathrm{dd}, J 4,10 \mathrm{~Hz}, \alpha \mathrm{H})$, $6.97(2 \mathrm{H}, \mathrm{d}, J 8 \mathrm{~Hz}, o-\mathrm{ArH}), 7,00(2 \mathrm{H}, \mathrm{d}, J 8 \mathrm{~Hz}, m-\mathrm{ArH}), 7.56\left(1 \mathrm{H}, \mathrm{s}, \mathrm{Im}^{2} \mathrm{H}\right) ;{ }^{13} \mathrm{C} \mathrm{NMR}(600$ $\left.\mathrm{MHz}, \mathrm{CD}_{3} \mathrm{OD}\right): \delta_{\mathrm{C}} 21.1\left(\mathrm{ArCH}_{3}\right), 28.7\left(\mathrm{Boc}_{-} \mathrm{CH}_{3}\right), 31.1(\beta \mathrm{C}), 32.7\left(\mathrm{NCH}_{3}\right), 41.2\left(\mathrm{SCH}_{2}\right), 55.9$ $(\alpha \mathrm{C}), 79.5$ (Boc-t-C), 129.7 (o-Ar), $129.8\left(\mathrm{Im}^{5} \mathrm{C}\right), 129.9$ (m-Ar), 134.5 (p-Ar), 137.5 (i-Ar), $139.3\left(\mathrm{Im}^{4} \mathrm{C}\right), 156.9\left(\mathrm{Im}^{2} \mathrm{C}\right), 161.4\left(\right.$ Boc-C=O), $178.0\left(\mathrm{CO}_{2} \mathrm{H}\right) ;$ HRMS m/z $[\mathrm{M}+\mathrm{H}]+$ Calcd: 406.1800, Found: 406.1794.

(2S)-2-Amino-3-(1-methyl-4-sulfanyl-1H-imidazol-5-yl)propanoic acid (6). To a stirred solution of $5(82 \mathrm{mg}, 0.2 \mathrm{mmol})$ in anisole $(0.5 \mathrm{~mL})$ cooled to $0{ }^{\circ} \mathrm{C}$ was added a solution of mercury(II) trifluoroacetate $(173 \mathrm{mg}, 0.4 \mathrm{mmol})$ in trifluoroacetic acid $(5 \mathrm{~mL})$. The mixture was stirred at $0{ }^{\circ} \mathrm{C}$ for $2 \mathrm{~h}$, concentrated under vacuum, taken up in water $(3 \mathrm{~mL})$, and washed with diethyl ether $(3 \times 1 \mathrm{~mL}) . \mathrm{H}_{2} \mathrm{~S}$ was bubbled into the aqueous solution for $15 \mathrm{~min}$, the suspension was filtered through Celite and the filtrate was concentrated under vacuum to give the di(trifluoroacetate) salt of the title compound $6(67 \mathrm{mg}, 78 \%)$ as a pale green glass: ${ }^{1} \mathrm{H}$ NMR $\left(600 \mathrm{MHz}, 5 \% \mathrm{D}_{2} \mathrm{O}\right): \delta_{\mathrm{H}} 3.51(1 \mathrm{H}, \mathrm{dd}, J 8,16 \mathrm{~Hz}, \beta \mathrm{H}), 3.67(1 \mathrm{H}, \mathrm{dd}, J 8,16 \mathrm{~Hz}, \beta \mathrm{H}), 4.51(1 \mathrm{H}$, dd, $J 8 \mathrm{~Hz}, \alpha \mathrm{H}), 4.65\left(3 \mathrm{H}, \mathrm{s}, \mathrm{NCH}_{3}\right), 8.75\left(1 \mathrm{H}, \mathrm{s}, \mathrm{Im}^{2} \mathrm{H}\right) ;{ }^{13} \mathrm{C} \mathrm{NMR}\left(600 \mathrm{MHz}, 5 \% \mathrm{D}_{2} \mathrm{O}\right): \delta_{\mathrm{C}} 26.4$ $(\beta \mathrm{C}), 36.8\left(\mathrm{NCH}_{3}\right), 53.7(\alpha \mathrm{C}), 122.0\left(\mathrm{Im}^{5} \mathrm{C}\right), 133.8\left(\mathrm{Im}^{4} \mathrm{C}\right), 139.0\left(\mathrm{Im}^{2} \mathrm{C}\right), 172.7\left(\mathrm{CO}_{2} \mathrm{H}\right)$; UV $\left(\lambda_{\max }, \mathrm{nm}, \mathrm{H}_{2} \mathrm{O}\right) 237,278$ (shoulder) ${ }^{19}$; HRMS $\mathrm{m} / z$ [M+H] ${ }^{+}$Calcd: 202.0650, Found: 202.0654.

(2S,2'S)-3,3'-[Disulfanediylbis(1-methyl-1H-imidazol-4,5-diyl)]bis(2-aminopropanoic acid) (7). For analytical purposes compound $6(50 \mathrm{mg}, 0.1 \mathrm{mmol})$ was taken up in water $(10 \mathrm{~mL})$, then air was bubbled through the solution for $1 \mathrm{~h}$. NMR studies showed that the oxidation of 6 proceeded quantitatively. Freeze-drying the solution yielded the tetra(trifluoroacetate) salt of 7 (49.4 mg, 99\%): ${ }^{1} \mathrm{H} \mathrm{NMR}\left(600 \mathrm{MHz}, 5 \% \mathrm{D}_{2} \mathrm{O}\right): \delta_{\mathrm{H}} 2.35(1 \mathrm{H}, \mathrm{dd}, J 7.5,16 \mathrm{~Hz}, \beta \mathrm{H}), 2.53(1 \mathrm{H}, \mathrm{dd}$, $J$ 7.5, $16 \mathrm{~Hz}, \beta \mathrm{H}), 3.38(1 \mathrm{H}, \mathrm{dd}, J 7.5 \mathrm{~Hz}, \alpha \mathrm{H}), 3.63\left(3 \mathrm{H}, \mathrm{s}, \mathrm{NCH}_{3}\right), 7.72\left(1 \mathrm{H}, \mathrm{s}, \mathrm{Im}^{2} \mathrm{H}\right) ;[\alpha]_{\mathrm{D}}{ }^{20}$ $+74\left(c 0.5,0.1 \mathrm{M} \mathrm{HCl}_{(\mathrm{aq})}\right) ;{ }^{19} \mathrm{UV}\left(0.1 \mathrm{M} \mathrm{HCl}_{(\mathrm{aq})}\right) \lambda_{\max } 257 \mathrm{~nm} ; \mathrm{HRMS} \mathrm{m/z}[\mathrm{M}+\mathrm{H}]^{+} \mathrm{Calcd}:$ 400.0987, Found: 400.0988 . 


\section{Supplementary Material Available}

The NMR spectra of compounds 1-7 can be found in the Supplementary Material section of this article.

\section{Acknowledgements}

This research was funded by TÁMOP 4.2.1.B-09/1/KMR and OTKA T 73804. The authors report no conflict of interest.

\section{References}

1. Palumbo, A.; d'Ichia, M.; Misuraca, G.; Prota, G. Tetrahedron Lett. 1982, 23, 3207-3208. http://dx.doi.org/10.1016/S0040-4039(00)88597-4

2. Palumbo, A.; Misuraca, G.; d'Ischia, M.; Donaudy, F.; Prota, G. Comp. Biochem. Physiol. 1984, 78B, 81-83.

3. Turner, E.; Somers, C. E.; Shapiro, B. J. Biol. Chem. 1985, 260, 13163-13171.

4. Turner, E.; Klevit, R.; Hager, L. J.; Shapiro, B. M. Biochemistry 1987, 26, 4028-4036. http://dx.doi.org/10.1021/bi00387a043

5. Weaver, K. H.; Rabenstein, D. L. J. Org. Chem. 1995, 60, 1904-1907.

6. Mirzahosseini, A.; Orgován, G.; Hosztafi, S.; Noszál, B. Anal. Bioanal. Chem. 2014, 406, 2377-2387.

http://dx.doi.org/10.1007/s00216-014-7631-0

7. Marjanovic, B.; Simic, M. G.; Jovanovic, S. V. Free Radical Biol. Med. 1995, 18, 679-685. http://dx.doi.org/10.1016/0891-5849(94)00186-N

8. Zoete, V.; Bailly, F.; Vezin, H.; Teissier, E.; Duriez, P.; Fruchart, J. C.; Catteau, J. P.; Bernier, J. L. Free Radical Res. 2000, 32, 515-524. http://dx.doi.org/10.1080/10715760000300521

9. Turner, E.; Hager, L. J.; Shapiro, B. M. Science 1988, 242, 939-941. http://dx.doi.org/10.1126/science.3187533

10. Holler, T. P.; Hopkins, P. B. Biochemistry 1990, 29, 1953-1961. http://dx.doi.org/10.1021/bi00459a042

11. Spies, H. S. C.; Steenkamp, D. J. Eur. J. Biochem. 1994, 224, 203-213. http://dx.doi.org/10.1111/j.1432-1033.1994.tb20013.x

12. Ariyanayagam, M. R.; Fairlamb, A. H. Mol. Biochem. Parasit. 2001, 115, 189-198. http://dx.doi.org/10.1016/S0166-6851(01)00285-7

13. Ondarza, R. N.; Iturbe, A.; Hernández, E.; Hurtado, G. Appl. Biochem. 2002, 36, 195-204. http://dx.doi.org/10.1042/BA20010102 
14. Ondarza, R. N.; Iturbe, A.; Hernández, E.; Hurtado, G. Appl. Biochem. 2003, 37, 195-204. http://dx.doi.org/10.1042/BA20020082

15. Krauth-Siegel, R. L.; Leroux, A. E. Antioxid. Redox Sign. 2012, 17, 583-607. http://dx.doi.org/10.1089/ars.2011.4392

16. Schmidt, A.; Krauth-Siege, R. L. Curr. Top. Med. Chem. 2002, 2, 1239-1259. http://dx.doi.org/10.2174/1568026023393048

17. Steenkamp, D. J. Life 2002, 53, 243-248.

18. Holler, T. P.; Salpenstein, A.; Turner, E.; Klevit, R. E.; Shapiro, B. M.; Hopkins, P. B. J. Org. Chem. 1987, 52, 4421-4423. http://dx.doi.org/10.1021/jo00228a060

19. Holler, T. P.; Ruan, F.; Salpenstein, A.; Hopkins, P. B. J. Org. Chem. 1989, 54, 4570-4574. http://dx.doi.org/10.1021/jo00280a022

20. Ohba, M.; Mukaihira, T.; Fujii, T. Heterocycles 1992, 33, 21-26. http://dx.doi.org/10.3987/COM-91-S2

21. Ohba, M.; Makaihira, T.; Fujii, T. Chem. Pharm. Bull 1994, 42, 1784-1790. http://dx.doi.org/10.1248/cpb.42.1784

22. Abdo, M.-R.; Joseph, P.; Boigegrain, R.-A.; Liautard, J.-P.; Montero, J.-L.; Köhler, S.; Winum, J.-Y. Bioorg. Med. Chem. 2007, 15, 4427-4433. http://dx.doi.org/10.1016/j.bmc.2007.04.027

23. Ito, S. J. Org. Chem. 1985, 50, 3636-3638. http://dx.doi.org/10.1021/jo00219a044

24. Van Der Berge, E.; Robiette, R. J. Org. Chem. 2013, 78, 12220-12223. http://dx.doi.org/10.1021/jo401978b

25. Jain, R.; Avramovitch, B.; Cohen, L. A. Tetrahedron 1998, 54, 3235-3242. http://dx.doi.org/10.1016/S0040-4020(98)00068-4

26. Cerezo, V.; Amblard, M.; Martinez, J.; Vardié, P.; Planas, M.; Feliu, L. Tetrahedron 2008, 64, 10538-10545. http://dx.doi.org/10.1016/j.tet.2008.08.077

27. European Pharmacopoeia, 7th Edn; European Council: Strasbourg, 2013.

28. Colombo, R.; Colombo, F. J. Chem. Soc., Perkin Trans. 1. 1985, 1811-1815. http://dx.doi.org/10.1039/p19850001811

29. Zaramella, S.; Heinonen, P.; Yeheskiely, E.; Strömberg, R. J. Org. Chem. 2003, 68, 75217523.

http://dx.doi.org/10.1021/jo0347967 